G. Leger and E. Luks.

Nagoya Math. J.

Vol. 44 (1971), $39-50$

\title{
ON DERIVATIONS AND HOLOMORPHS OF NILPOTENT LIE ALGEBRAS
}

\author{
G. Leger and E. Luks ${ }^{1)}$
}

\section{§1. Introduction}

A linear Lie algebra is called toroidal if it is abelian and consists of semi-simple transformations. The maximum, $t(L)$, of the dimensions of the toroidal subalgebras of the derivation algebra, $\Delta(L)$, is an invariant of $L$. This paper is mainly concerned with the relation between the magnitude of $t(L)$ for nilpotent $L$ and the structures of $L$ and $\Delta(L)$.

Now, if $L$ is quasi-cyclic (in particular, if $L^{3}=0$ ) then $t(L) \geq 1$. We discuss a class of metabelian algebras in which the equality holds. The holomorphs of these algebras have a remarkably simple structure and their study lead to the discovery of non-isomorphic nilpotent Lie algebras with isomorphic holomorphs and even isomorphic derivation algebras. The question of whether or not Lie algebras are determined by their holomorphs was first studied by Schenkman in [5]. He showed that free nilpotent Lie algebras are so determined but the general question was unsettled until now.

It was conjectured in [4] that if $t(L)=1$ then $\Delta(L)$ is solvable. We show (Theorem 2.2) that this is correct provided $L$ has a non-singular derivation and give an example showing that this latter restriction is needed. In section 3 we investigate briefly the restrictions imposed on a nilpotent Lie algebra by a requirement that most of its ideals be characteristic. If every ideal of $L$ containing $L^{2}$ is characteristic then $t(L) \leq 1$. Also, if every ideal of a metabelian Lie algebra over an algebraically closed field is characteristic then $L$ can only be one dimensional.

If $L$ is nilpotent and $L=U+U^{2}+\cdots+U^{k}$ ( $\dot{+}$ denotes vector space direct sum), where $U$ is a subspace of $L$ and $U^{i}=\left[U, U^{i-1}\right]$, then $L$ is called quasi-cyclic. If the ground field has characteristic 0 , a nilpotent Lie algebra is quasi-cyclic if and only if there is a subspace $U$ with $L=U+L^{2}$ such that

Received July 13, 1970

1) The authors are grateful to the National Science Foundation for its support via grants GP-11162 and GP-11163. 
the identity map $I_{U}$ extends to a derivation of $L$. This is not the case in characteristic $p>0$. However, the extension of $I_{U}$ to a derivation occurs often and, when it exists, we still write $I_{U}$ for this (unique) extension.

\section{§2. Solvability of $\Delta(L)$.}

2.1 Remark: A nilpotent Lie algebra is abelian if and only if $L / L^{3}$ is abelian.

2.2 Theorem. Let $L$ be nilpotent, non-abelian over a field of characteristic 0 . Let $D$ in $\Delta(L)$ induce a non-singular derivation of $L / L^{3}$. Then $D$ is not contained in any simple three-dimensional subalgebra of $\Delta(L)$.

Proof. We may assume, without loss of generality, that the ground field is algebraically closed. Let $D$ induce the non-singular derivation $\bar{D}$ on $L / L^{3} . \quad \bar{D}_{s}$ (the semi-simple component of $\bar{D}$ ) is induced by the semi-simple component $D_{s}$ of $D$ and $\bar{D}$ is non-singular if and only if $\bar{D}_{s}$ is. If $D$, and therefore also $D_{s}$ (a semi-simple Lie algebra is algebraic) is contained in a three-dimensional simple subalgebra $S$ of $\Delta(L)$ then $\bar{D}$ (and therefore also $\bar{D}_{s}$ ) is contained in the three-dimensional subalgebra $\bar{S}$ of $\Delta\left(L / L^{3}\right)$ induced by $S$, for the restriction to $S$ of the homomorphism $\Delta(L) \rightarrow \Delta\left(L / L^{3}\right)$ induced by the natural map $L \rightarrow L / L^{3}$ is injective since $L$ is nilpotent. This means that $L / L^{3}$ has a non-singular semi-simple derivation contained in a three-dimensional simple subalgebra of its derivation algebra. Thus we may assume that $L^{3}=0$ and that $D$ is semi-simple. Multiplying $D$, if necessary, by a nonzero scalar we may choose $E, F$, in $S$ so that $D, E, F$ is a basis of $S$ and so that

$$
[E, D]=2 E \quad[F, D]=-2 F \quad[E, F]=D .
$$

Now 0 is not a characteristic root of $D$ so the discussion on page 84 of [3] shows that all of the characteristic roots of $D$ are odd integers. Let $x_{1}, \cdots, x_{n}$ be a basis of $L$ consisting of characteristic vectors of $D$, say $D x_{i}=\rho_{i} x_{i}$ with the $\rho_{i}$ odd integers. Then $D\left[x_{i}, x_{j}\right]=\left[D x_{i}, x_{j}\right]+\left[x_{i}, D x_{j}\right]=$ $\left(\rho_{i}+\rho_{j}\right)\left[x_{i}, x_{j}\right]$ so that $\left[x_{i}, x_{j}\right]$ is an eigenvector of $D$ with corresponding characteristic root $\rho_{i}+\rho_{j}$. But $\rho_{i}+\rho_{j}$ is even whence $\left[x_{i}, x_{j}\right]=0$ and $L$ must be abelian.

2.3 Remark: A derivation as in 2.2 may be contained in a simple subalgebra of $\Delta(L)$ of dimension greater than 3 . Consider a free nilpotent 
Lie algebra $L$ generated by an $n$-dimensional vector space $U$ with $n>2$. Every element of $\operatorname{Hom}(U, U)$ extends uniquely to a derivation of $L$; and amongst these those of trace 0 form a simple subalgebra of $\Delta(L)$. Over an infinite base field some of these are non-singular.

2.4 Theorem. If $L$ is a Lie algebra over a ground field of characteristic 0 with $t(L)=1$ and if $L$ has a non-singular derivation then $\Delta(L)$ is solvable.

Proof: As in 2.2 we may assume that the ground field is algebraically closed and that $L$ has a non-singular semi-simple derivation $D$. If $\Delta(L)$ were not solvable then $\Delta(L)$ would contain a simple subalgebra $S$. A Cartan subalgebra of $S$ would consist of semi-simple transformations and so, since $t(L)=1$, it follows that $S$ can be chosen so that $D$ is in $S$. Now from the classification of simple Lie algebras it follows that $S$ has dimension 3. The result follows from 2.2 .

\section{§3. Characteristic ideals and $t(L)$.}

3.1 Proposition. Let $L$ be a nilpotent Lie algebra over a perfect field $F$ and let $D$ be a derivation of $L$ which induces the identity on $L / L^{2}$. Then there exists a subspace $U$ of $L$ such that $L=U+L^{2}$ and the identity map $I_{U}$ extends to a derivation of $L$.

Proof: Since the ground field is perfect we may write $D=S+N$ where $S$ is semi-simple, $N$ is nilpotent and $S$ is a derivation of $L$. Since the transformation induced by $D$ on $L / L^{2}$ is semi-simple it is identical with the transformation induced by $S$. Thus if $U$ is a subspace of $L$ with $S U \subset U$, $L=U+L^{2}$ then $S$ induces the identity on $U$.

3.2 Remark: If $F$ has characteristic 0 and $L$ is as in 3.1 then $L$ is quasi-cyclic i.e., $L=U+U^{2}+\cdots+U^{k}$. However this need not be the case if the characteristic is $p>0$. To see this let $L_{1}=U_{1}+U^{2}+\cdots+U_{1}{ }^{k}$ and $L_{2}=U_{2}+U_{2}{ }^{2}+\cdots+U_{2}{ }^{k+p}$ be quasi-cyclic Lie algebras over a field of characteristic $p>0$, where we assume $k>1$ and $U_{1}{ }^{k} \neq 0, U_{2}{ }^{k+p} \neq 0$. Choose a non-zero $x_{1}$ in $U_{1}{ }^{k}$ and a non-zero $x_{2}$ in $U_{2}{ }^{k+p}$ and let $L=L_{1} \oplus L_{2} / J$ where $J$ is the ideal of $L_{1} \oplus L_{2}$ generated by $\left(x_{1}, x_{2}\right)$. This $L$ is not quasicyclic. However $L=U+U^{2}+\cdots+U^{k+p}$ where $U$ is the image of $U_{1} \oplus U_{2}$ 
in $L$ and the map which is $j$ times the identity on each power $U^{j}$ is welldefined and is a derivation of $L$.

3.3 Remark: If $L$ is a nilpotent Lie algebra the collection of derivations $D$ such that $D L \subset L^{2}$ is a nilpotent ideal of $\Delta(L)$.

3.4 Proposition: Let $L$ be a nilpotent Lie algebra over any field $F$. Suppose that every ideal of $L$ which contains $L^{2}$ is characteristic. Then every derivation induces a scalar multiple of the identity on $L / L^{2}$.

Proof: Take $\delta$ in $\Delta(L)$. For any $x$ in $L$, with $x$ not in $L^{2}$, we have $\delta x \equiv \lambda(x) x \bmod L^{2}$ with $\lambda(x)$ in $F$ since the ideal $\left(x, L^{2}\right)$ is characteristic. If $x$ and $y$ are linearly independent and are not in $L^{2}$ then $\lambda(x)=\lambda(y)$ since $\left(x-y, L^{2}\right)$ is characteristic.

3.5 Corollary: Let $L$ be nilpotent and such that every ideal of $L$ which contains $L^{2}$ is characteristic. If the ground field is perfect then $t(L) \leq 1$. Further, every ideal of $L$ situated between powers of $L$ is characteristic.

3.6 Remark: If $L^{3}=0$ the elements of $\operatorname{Hom}\left(L, L^{2}\right)$ which map $L^{2}$ to 0 are derivations of $L$.

3.7 Lemma: Let $L$ be a Lie algebra with $L^{3}=0$ and $\operatorname{dim} L^{2} \geq 2$ over an algebraically closed field. Then there exists an $x$ in $L$ not in $L^{2}$ with $[x, L] \neq L^{2}$.

Proof. Assume first that $\operatorname{dim} L^{2}=2$.

Let $x_{1}, \cdots, x_{m}, a, b$ be a basis of $L$ with $a, b$ a basis of $L^{2}$. Let $\left[x_{i}, x_{j}\right]=\alpha_{i j} a+\beta_{i j} b$ and put $A=\left(\alpha_{i j}\right), B=\left(\beta_{i j}\right)$. Choose $\lambda$ in the ground field such that $A-\lambda B$ is singular and let $r=\left(r_{1}, r_{2}, \cdots, r_{m}\right)$ be a non-trivial solution of $r(A-\lambda B)=0$. Then $\left[\sum r_{i} x_{i}, L\right] \subseteq(\lambda a+b) \varsubsetneqq L^{2}$. If $\operatorname{dim} L^{2}=s>2$ the problem reduces to the above by factoring out an ideal of dimension $s-2$ contained in $L^{2}$.

3.8 Proposition: Let $L$ be a Lie algebra with $L^{3}=0$ over an algebraically closed field. If every ideal is characteristic then $\operatorname{dim} L=1$.

Proof: We shall show that $L^{2}=0$. Then $L$ abelian with every ideal characteristic forces $\operatorname{dim} L=1$.

First suppose $\operatorname{dim} L^{2} \geq 2$. 3.7 gives us an $x$ not in $L^{2}$ with $[x, L] \neq L^{2}$. But then the ideal $(x)+[x, L]$ is not characteristic. 
If $\operatorname{dim} L^{2}=1$. Write $L=V+(z)$. For $v_{1}, v_{2}$ in $V,\left[v_{1}, v_{2}\right]=\mu\left(v_{1}, v_{2}\right) z$ where $\mu$ is a skew symmetric bilinear form on $V$. If the rank of $\mu$ were less than $\operatorname{dim} V$ then $L$ would have an abelian direct summand, which would be a non-characteristic ideal. Thus we may choose a basis $v_{1}, \cdots, v_{s}$, $w_{1}, \cdots, w_{s}$ for $V$ so that $\left[v_{i}, w_{j}\right]=\delta_{i j} z$. There is a derivation mapping $v_{1} \rightarrow w_{1}, w_{1} \rightarrow v_{1}$ and other basis elements to 0 . But then the ideal $\left(v_{1}\right)+L^{2}$ is not characteristic.

3.9 The hypothesis $L^{3}=0$ cannot be removed from Proposition 3.8 as seen from the example, $L$, of a characteristically nilpotent Lie algebra given by Dixmier-Lister [2].

For the reader's convenience we give the multiplication table; the example has a basis $x_{1}, \cdots, x_{8}$ with $\left[x_{1}, x_{2}\right]=x_{5},\left[x_{1}, x_{3}\right]=x_{6},\left[x_{1}, x_{4}\right]=x_{7}$, $\left[x_{1}, x_{5}\right]=-x_{8},\left[x_{2}, x_{3}\right]=x_{8},\left[x_{2}, x_{4}\right]=x_{6},\left[x_{2}, x_{6}\right]=x_{7},\left[x_{3}, x_{4}\right]=-x_{5},\left[x_{3}, x_{5}\right]=$ $-x_{7},\left[x_{4}, x_{6}\right]=-x_{8}$. As shown in $[2], \Delta(L)=N_{0}+I(L)$ where $N_{0}=\left\{D \mid D L \subset L^{3}\right\}$ and $I(L)$ denotes the inner derivations. It is an easy matter to check that every ideal of this example is situated between powers of the algebra and thus, since the derivation algebra has the above structure, every ideal is characteristic.

3.10 One may ask if every characteristically nilpotent Lie algebra has only characteristic ideals. An example showing that this is not the case is constructed as follows: Start with the example, $L$, given in Bourbaki [1, page 123]. Here $L=\left(x_{1}, \cdots, x_{8}\right)$ with

$$
\begin{array}{llll}
{\left[x_{1}, x_{2}\right]=x_{3}} & {\left[x_{1}, x_{3}\right]=x_{4}} & {\left[x_{1}, x_{4}\right]=x_{5},} & {\left[x_{1}, x_{5}\right]=x_{6}} \\
{\left[x_{1}, x_{6}\right]=x_{8}} & {\left[x_{1}, x_{7}\right]=x_{8}} & {\left[x_{2}, x_{3}\right]=x_{5},} & {\left[x_{2}, x_{4}\right]=x_{6}} \\
{\left[x_{2}, x_{5}\right]=x_{7}} & {\left[x_{2}, x_{6}\right]=2 x_{8}} & {\left[x_{3}, x_{4}\right]=-x_{7}+x_{8} \quad\left[x_{3}, x_{5}\right]=-x_{8}}
\end{array}
$$

other products 0 . This algebra also has only characteristic ideals. Now let $H$ be the nine-dimensional algebra $\left(x_{0}\right)+L$ with $\left[x_{0}, x_{1}\right]=x_{7},\left[x_{0}, x_{i}\right]=0$, $i \neq 1$.

A calculation much like the one indicated in Bourbaki [op. cit.] shows that $H$ is characteristically nilpotent. Note however that the mapping which sends $x_{1} \rightarrow x_{0}$ and $x_{i} \rightarrow 0$ for $i \neq 1$ is a derivation of $H$ and thus $\Delta(H) H \nsubseteq H^{2}$. Thus $H$ has a non-characteristic ideal, e.g. $\left(x_{1}\right)+H^{2}$.

\section{§4. $L^{3}=0$ and holomorphs.}

If $L^{3}=0$ and $L=U+U^{2}$ then every element $h$ of $\operatorname{Hom}\left(U, U^{2}\right)$ extends 
uniquely to an element, still denoted by $h$, of $\Delta(L)$ such that $h\left(U^{2}\right)=0$. By this means $\operatorname{Hom}\left(U, U^{2}\right)$ is identified with an abelian subalgebra of $A(L)$. Further $I_{U}$ extends to a derivation of $L$ also denoted by $I_{U}$. One naturally asks if an algebra with $L^{3}=0$ can have a derivation algebra no larger than $\left(I_{U}\right)+\operatorname{Hom}\left(U, U^{2}\right)$. We shall give two non-isomorphic examples over any fixed ground field of characteristic 0 .

Let $L_{1}$ have a basis $x_{1}, \cdots, x_{9}$ with the multiplication table

$$
\begin{array}{llll}
{\left[x_{1}, x_{2}\right]=x_{6}} & {\left[x_{1}, x_{3}\right]=x_{7}} & {\left[x_{1}, x_{5}\right]=x_{8}} & {\left[x_{2}, x_{3}\right]=x_{9}} \\
{\left[x_{2}, x_{4}\right]=x_{8}} & {\left[x_{2}, x_{5}\right]=x_{7}} & {\left[x_{3}, x_{4}\right]=x_{6}} & {\left[x_{4}, x_{5}\right]=x_{9}}
\end{array}
$$

with $\left[x_{i}, x_{j}\right]=0$ for $i<j$ if it is not in the above list.

Let $U$ be the subspace $\left(x_{1}, x_{2}, x_{3}, x_{4}, x_{5}\right), U^{2}=\left(x_{6}, x_{7}, x_{8}, x_{9}\right)$ and computation will show that $\Delta\left(L_{1}\right)=\left(I_{U}\right)+\operatorname{Hom}\left(U, U^{2}\right)$.

Let $L_{2}$ have a basis $y_{1}, \cdots, y_{9}$ with the multiplication table

$$
\begin{array}{llll}
{\left[y_{1}, y_{2}\right]=y_{6}} & {\left[y_{1}, y_{3}\right]=y_{7}} & {\left[y_{2}, y_{3}\right]=y_{9}} & {\left[y_{2}, y_{4}\right]=y_{8}} \\
{\left[y_{2}, y_{5}\right]=y_{7}} & {\left[y_{3}, y_{4}\right]=y_{6}} & {\left[y_{3}, y_{5}\right]=y_{8}} & {\left[y_{4}, y_{5}\right]=y_{9}}
\end{array}
$$

with $\left[y_{i}, y_{j}\right]=0$ for $i<j$ if it is not in the above list.

Let $V=\left(y_{1}, y_{2}, y_{3}, y_{4}, y_{5}\right), \quad V^{2}=\left(y_{6}, y_{7}, y_{8}, y_{9}\right)$ and again computation will show that $\Delta\left(L_{2}\right)=\left(I_{V}\right)+\operatorname{Hom}\left(V, V^{2}\right)$.

To see that $L_{1}$ is not isomorphic to $L_{2}$ verify that the rank of the linear transformation ad $x$ is either zero or is greater than or equal to 3 for all $x$ in $L_{1}$ while ad $y_{1}$ has $\operatorname{rank} 2$ in $L_{2}$.

We shall show that these algebras have isomorphic holomorphs and to this end we introduce a type of Lie algebras as follows: Let $m, n$ be nonnegative integers let $U, V$ be vector spaces of dimensions $m, n$ respectively over the same field of characteristic $\neq 2$. Form an algebra $H\langle U, V\rangle$ whose underlying vector space is $(\theta)+\operatorname{Hom}(U, V)+U+V$ where $(\theta)$ is a onedimensional space. Make $H\langle U, V\rangle$ into a Lie algebra so that

$$
\begin{aligned}
& {[h, u]=h(u)} \\
& {[\theta, h]=h} \\
& {[\theta, u]=u} \\
& {[\theta, v]=2 v \quad \text { for all } h \text { in } \operatorname{Hom}(U, V), \text { all } u \text { in } U}
\end{aligned}
$$

and all $v$ in $V$ while 
$[\operatorname{Hom}(U, V), \operatorname{Hom}(U, V)]=[\operatorname{Hom}(U, V), V]$

$$
=[U, U]=[U, V]=[V, V]=0 .
$$

We shall denote an isomorph of the above algebra by $H\langle m, n\rangle$ whenever the spaces $U$ and $V$ do not require explication.

4.1 Proposition: $H\langle m, n\rangle$ has the following properties:

1) If $J$ is a proper ideal of $H\langle m, n\rangle$ then $J$ is nilpotent.

2) If $S$ is any subalgebra of $H\langle m, n\rangle$ then the maximal nilpotent ideal of $S$ has codimension in $S \leq 1$.

Proof: Suppose $J$ is a non-nilpotent ideal of $H\langle U, V\rangle$. Then there is an $x$ in $J$ of the form $x=\theta+h+u+v$ where $h$ is in $\operatorname{Hom}(U, V), \quad u$ in $U, v$ in $V$. But $[\theta, x]=h+u+2 v$ so $\theta-v$ is in $J$. Now $[\theta, \theta-v]=-2 v$ so that $v$, and therefore $\theta$ is in $J$. This means $J \supseteq[\theta, H\langle U, V\rangle]=\operatorname{Hom}(U, V)$ $+U+V$ so that $J=H\langle U, V\rangle$. 2) is clear.

4.2 Theorem: Let $L$ be a Lie algebra over a field of characteristic $\neq 2$ with holomorph $H(L)$ and derivation algebra $\Delta(L) . H(L)$ is isomorphic to $H\langle m, n\rangle$ for some $m, n$ if and only if

1) $L$ is nilpotent with $L^{3}=0$

2) If $L=U \dot{+} U^{2}$ then $\Delta(L)=\left(I_{U}\right)+\operatorname{Hom}\left(U, U^{2}\right)$.

Proof: Suppose $H(L) \approx H\langle m, n\rangle$. We may assume $\operatorname{dim} L>1 . \quad L$ is a proper ideal of its holomoph and thus, by 4.1, $L$ is nilpotent. Furthermore $L^{3}=0$ since the maximal nilpotent ideal of $H\langle m, n\rangle$ has that property. Now let $L=U+U^{2}$. As noted $\Delta(L) \supseteq\left(I_{U}\right)+\operatorname{Hom}\left(U, U^{2}\right)$.

Next we assert that $L$ has no non-zero abelian direct summand. For suppose $L=L_{1} \oplus A$, where $A$ is a non-zero abelian ideal. $I_{A}$ extends to a derivation of $L$, also denoted by $I_{A}$, such that $I_{A}\left(L_{1}\right)=0$. Since $I_{A}$ and $I_{U}$ are commuting, linearly independent, non-nilpotent derivations of $L$, the subspace $\left(I_{A}\right)+\left(I_{U}\right)+L$ of $H(L)$ is a subalgebra violating 2) of 4.1 .

Again using 2) of 4.1, $A(L)=\left(I_{U}\right)+N$, where $N$ is the maximal nilpotent ideal of $\Delta(L)$. If $D$ is in $N$ then some power of $a d_{\Delta(L)}(D)$ annihilates $\Delta(L)$ and hence also the ideal of inner derivations. This means that some power of $D$ maps $L$ into the center of $L$ which, since $L$ has no abelian direct summands, is contained in $L^{2}$. Then some higher power of $D$ annihilates $L$. Thus $N$ consists of nilpotent derivations and therefore $N+L$ is a nil- 
potent ideal of $H(L)$. It follows that $(N+L)^{3}=0$ and therefore that the square of every derivation in $N$ is 0 .

Take $D$ in $N, u$ in $U$. Let $v=D u$. If $v$ were not in $U^{2}$ then $u$ and $v$ would be independent since $D$ is a nilpotent linear transformation. Then one could choose $h$ in $\operatorname{Hom}\left(U, U^{2}\right)$ such that $h(u)=0, h(v) \neq 0$. But $D+h$ would be a derivation of $N$ with $(D+h)^{2} \neq 0$ which is impossible. Thus $N=\operatorname{Hom}\left(U, U^{2}\right)$.

Conversely suppose 1) and 2) hold. Then $H(L)=\left(I_{U}\right)+\operatorname{Hom}\left(U, U^{2}\right)+$ $U+U^{2} . \quad$ (Note that we are not finished; $H(L)$ is not $H\left\langle U, U^{2}\right\rangle$ since $[U, U] \neq 0$.) Put $V=\left\{u-\frac{1}{2} D_{u} \mid u\right.$ in $\left.U\right\}, W=U^{2}$. Then $[V, V]=[V, W]=0$ and $\left[I_{U}, v\right]=v$ for all $v$ in $V$. For $h$ in $\operatorname{Hom}\left[U, U^{2}\right],\left[h, u-\frac{1}{2} D_{u}\right]=h(u)$ so we may identify $\operatorname{Hom}\left(U, U^{2}\right)$ with $\operatorname{Hom}(V, W)$. Thus $H(L)=\left(I_{V}\right)+\operatorname{Hom}(V, W)+V$ $+W=H(V, W)$.

4.3 Corollary. If $H(L) \approx H\langle m, n\rangle$ then $\operatorname{dim} L=m+n$ and $\operatorname{dim} L^{2}=n$.

4.4 Corollary. For $\operatorname{dim} L>1, H(L) \approx H\langle m, n\rangle$ if and only if $L^{3}=0$ and the algebra of outer derivations, $\Delta(L) / I(L)$, is isomorphic to an algebra of the form $(I)+B$ where $B$ is an abelian ideal of dimension $m(n-1)$ and $[I, b]=b$ for all $b$ in $B$.

The above results coupled with the examples given at the beginning of this section give us the following:

4.5 Theorem. There are non-isomorphic Lie algebras $L_{1}, L_{2}$ with $L_{1}^{3}=L_{2}^{3}=0$ and with $\Delta\left(L_{1}\right) \approx \Delta\left(L_{2}\right), \Delta\left(L_{1}\right) / I\left(L_{1}\right) \approx \Delta\left(L_{2}\right) / I\left(L_{2}\right), H\left(L_{1}\right) \approx H\left(L_{2}\right)$.

4.6 The examples above both have holomorph $H\langle 5,4\rangle$ and a reasonable question is whether or not $L^{3}=0$ and $t(L)=1$ forces the holomorph of $L$ to be an $H\langle m, n\rangle$. The answer is no and an example $L$ is given as follows:

Let $L$ have a basis $x_{1}, x_{2}, \cdots, x_{10}$ over a field of characteristic 0 with the multiplication table:

$$
\begin{aligned}
& {\left[x_{1}, x_{2}\right]=\left[x_{3}, x_{4}\right]=\left[x_{5}, x_{6}\right]=x_{7}} \\
& {\left[x_{1}, x_{3}\right]=\left[x_{2}, x_{5}\right]=x_{8}} \\
& {\left[x_{1}, x_{5}\right]=\left[x_{2}, x_{4}\right]=x_{9}} \\
& {\left[x_{2}, x_{3}\right]=\left[x_{4}, x_{5}\right]=x_{10} \text { where }\left[x_{i}, x_{j}\right]=0}
\end{aligned}
$$


if it does not appear in the list. Computation shows that $\Delta(L)$ is spanned by $I_{U}$ where $U=\left(x_{1}, x_{2}, \cdots, x_{6}\right), D$ with $D x_{5}=x_{6}$ and $D x_{i}=0$ if $i \neq 5$ and $\operatorname{Hom}\left(U, U^{2}\right)$. Clearly $t(L)=1$ however $\Delta(L) \neq\left(I_{U}\right)+\operatorname{Hom}\left(U, U^{2}\right)$ so that $H(L) \neq H\langle 6,4\rangle$ by Theorem 4.2 .

It is worth noting that our examples of Lie algebras with holomorphs $H(m, n)$ are "minimal". We shall show this in 4.9.

Let $V$ be the space of $m \times m$ matrices, $m>2$, over a field of characteristic $\neq 2, W$ the $\frac{m(m-1)}{2}$-dimensional subspace of skew-symmetric matrices. Define $\phi: V \rightarrow \operatorname{Hom}(W, W)$ by $\phi(B)(A)=B A+A B^{t}$ for $A$ in $W, B$ in $V$. Then $\phi$ is an injective linear transformation and $\phi(I)=2 I_{W}$.

Suppose $L=U+U^{2}$ with $u_{1}, \cdots, u_{m}$ a basis of $U, y_{1}, y_{2}, \cdots, y_{n}$ a basis of $U^{2}$ and $\left[u_{i}, u_{j}\right]=\sum_{k} a_{i j}^{k} y_{k}$. Let $X$ be the subspace of $W$ spanned by $\left(a_{i j}^{k}\right)$, $k=1,2, \cdots, n$. Note that $\operatorname{dim} X=n$ and

$$
\text { (*) } \quad 0 \leq n \leq \frac{m(m-1)}{2} .
$$

One may verify

4.7 Proposition. A map $T$ in $\operatorname{Hom}(U, U)$ extends to a derivation of $L$ iff $X$ is $\phi\left(t_{i j}\right)$ invariant, where $T u_{i}=\sum_{j} t_{i j} u_{j}$.

We shall need also the following lemma.

4.8 Lemma. Let $X$ be a subspace of a vector space $W, C$ a subspace of $\operatorname{Hom}(W, W)$ such that $C \cap \operatorname{Hom}_{X}(W, W)=\left(I_{W}\right)$ ( $T$ is in $\operatorname{Hom}_{X}(W, W)$ iff $\left.T(X) \subseteq X\right)$, then $\operatorname{dim} C \leq \operatorname{dim} X \cdot \operatorname{codim} X+1$.

Proof. Let $r=\operatorname{dim} W, s=\operatorname{dim} X, D=\operatorname{Hom}_{X}(W, W)$.

$$
\text { Then } \begin{aligned}
r^{2} & =\operatorname{dim}(\operatorname{Hom}(W, W)) \\
& \geq \operatorname{dim}(C+D) \\
& =\operatorname{dim} C+\operatorname{dim} D-\operatorname{dim}(C \cap D) \\
& =\operatorname{dim} C+r^{2}-r s+s^{2}-1 .
\end{aligned}
$$

The result follows.

We can now prove

4.9 Theorem. If $H(L)=H\langle m, n\rangle$ for some $L$ of dimension $>1$, then either $m=5$ with $4 \leq n \leq 6$ or $m \geq 6$ with $3 \leq n \leq \frac{m(m-1)}{2}-3$. In particular, dim $L \geq 9$. 
Proof. Suppose $H(L)=H\langle m, n\rangle . \quad$ By 4.3, $L=U+U^{2}, \operatorname{dim} U=m$, $\operatorname{dim} U^{2}=n$, and the only elements of $\operatorname{Hom}(U, U)$ which extend to derivations of $L$ are the scalar multiples of the identity.

It follows from 4.7 that

$$
\phi(V) \cap \operatorname{Hom}_{X}(W, W)=\left(I_{W}\right) .
$$

By computation, $L$ is not one of the two algebras with $m=2$ and then by 4.8

$$
m^{2} \leq\left(\frac{m(m-1)}{2}-n\right) n+1
$$

If $m=3$ or $4(*)$ and $(* *)$ cannot be satisfied simultaneously. If $m=5$, these inequalities yield $4 \leq n \leq 6$. Finally, if $m \geq 6,(* *)$ is not satisfied by the six solutions of $(*)$ not in the range $3 \leq n \leq \frac{m(m-1)}{2}-3$.

\section{§5. An answer to a conjecture concerning $t(L)$.}

If $L$ is an $H\langle m, m\rangle$ then Theorem 4.2 gives us the structure of $\Delta(L)$ and we see that $t(L)=1$ while the maximal nilpotent ideal of $\Delta(L)$ is Hom $\left(U, U^{2}\right)$ where $L=U \dot{+} U^{2}$. It seems natural to ask whether there exists a metabelian Lie algebra $L$ with maximal nilpotent ideal of $\Delta(L)=\operatorname{Hom}\left(U, U^{2}\right)$ and $t(L)>1$. We shall give an example of such an algebra with $t(L)=$ $\operatorname{dim} L / L^{2}$ (the maximum possible for $t(L)$ ).

The example $L$ has a basis $x_{1}, \cdots, x_{10}$ over a field of characteristic 0 with

$$
\begin{aligned}
& {\left[x_{1}, x_{3}\right]=x_{6} \quad\left[x_{2}, x_{4}\right]=x_{7}} \\
& {\left[x_{2}, x_{3}\right]=x_{8} \quad\left[x_{1}, x_{5}\right]=x_{9} \quad\left[x_{4}, x_{5}\right]=x_{10}}
\end{aligned}
$$

and $\left[x_{i}, x_{j}\right]=0$ for $i<j$ if it is not in this list.

Computation will verify that the derivations of this algebra have desired structure i.e., $t(L)=5$ and every nilpotent derivation maps $L$ into $L^{2}$.

Now we shall show that $L$ yields a counterexample to the conjecture $t(L) \leq t(L / J)+t(J)$ ([4], Remark 3, p. 640) for any ideal $J$ of $L$. To see this let $J$ be the ideal generated by

$z_{1}=x_{1}+x_{2}, \quad z_{2}=x_{1}+x_{3}, \quad z_{3}=x_{1}+x_{4}, \quad z_{4}=x_{5}-x_{4} . \quad$ First note that $\left[\left(z_{1}, \cdots, z_{4}\right),\left(z_{1}, \cdots, z_{4}\right)\right]=\left(x_{6}, \cdots, x_{10}\right)$ so that codim $J=1$ and therefore $t(L / J)=1$. To see that $t(J)=3$ (as least in characteristic 0 ) suppose $T$ is a 
linear transformation of $J$ such that $T z_{i}=\lambda_{i} z_{i}$ then $T$ is a derivation of $J$ if and only if $2 \lambda_{1}=\lambda_{2}-\lambda_{3}$. Now let $A=\left\{T \mid T z_{i}=\lambda_{i} z_{i} ; 2 \lambda_{1}=\lambda_{2}-\lambda_{3}\right\}$ and check easily that $A$ is a maximal toroidal subalgebra of $S(J)$ and has dimension 3. Thus $L$ yields the desired example since $5 \npreceq 1+3$.

\section{§6. Resolutions of some questions about $\Delta(\boldsymbol{L})$.}

In this section we shall resolve the following questions concerning the structure of derivation algebras of nilpotent Lie algebras:

6.1 Does there exists a nilpotent Lie algebra $L$ with $t(L)=1$ such that $\Delta(L)$ is not solvable? (See [4, p. 640] Remark 5).

6.2 Does there exist a nilpotent Lie algebra $L$ such that the radical of $\Delta(L)$ consists of nilpotent derivations but is not a direct summand of $L(L)$ ? (See [6, p. 214]).

6.3 Does there exist a directly indecomposable nilpotent Lie algebra $L$ which has a semi-simple derivation but no non-singular derivation?

6.4 Does there exist a nilpotent Lie algebra $L$ such that $\Delta(L)$ annihilates the center of $L$ but $L$ is not characteristically nilpotent? (The affirmative answer to this question shows that the claim added to Remark 5 in [4] is incorrect.)

We construct a Lie algebra $L$ which answers 6.1-6.4 in the affirmative.

We begin with any characteristically nilpotent Lie algebra $A$ such that $\{w$ in $A \mid[w, A] \subseteq Z(A)\} \subseteq A^{2}$ where $Z(A)$ is the center of $A$. Note that the algebra given by Dixmier-Lister [2] has this property. Let $V$ be a twodimensional vector space over the ground field of $A$ with basis $x, y$. Take a non-zero $z$ in $Z(A)$. Now let $L$ be the Lie algebra whose underlying vector space is the direct sum of $V$ and that of $A$. Products are defined in $L$ so that $[x, y]=z,[V, A]=0$, and the products in $A$ are as before.

6.5 Proposition. $\Delta(L)=S+N$ where $S$ is a simple three-dimensional subalgebra and $N$ is an ideal consisting of nilpotent derivations.

Proof. First note that any linear transformation $D$ in $\operatorname{Hom}(L, L)$ induces $D_{1}$ in $\operatorname{Hom}(L, V)$ and $D_{2}$ in $\operatorname{Hom}(L, A)$ such that $D(u)=D_{1}(u)+D_{2}(u)$ for all $u$ in $L$. Now if $D$ is in $\Delta(L)$ :

(1) $\left.D_{2}\right|_{A}$ is in $A(A)$.

(2) $\operatorname{trace}\left(\left.D_{1}\right|_{V}\right)=0$. 
Since $A^{2} \subset A$ and $[V, A]=0$, for $a, b$ in $A$ one has $D_{2}[a, b]=D[a, b]=[D a, b]+$ $[a, D b]=\left[D_{2} a, b\right]+\left[a, D_{2} b\right]$; hence (1). For (2), let $D_{1}(x)=\alpha x+\beta y, D_{1}(y)=$ $\gamma x+\delta y$. Then $D z=D[x, y]=(\alpha+\delta) z$. By (1) and the characteristic nilpotence of $A, \alpha+\delta=0$.

Any $T$ in $\operatorname{Hom}(V, V)$ extends to a unique $T^{*}$ in $\operatorname{Hom}(L, L)$ such that $T^{*}(v)=T(v)$ for $v$ in $V$ and $T^{*}(A)=0$. Let $S=\left\{T^{*} \mid T\right.$ in $\operatorname{Hom}(V, V)$, trace $T=0$. One sees easily that $S \subset \Delta(L)$ and that $S$ is simple of dimension three.

$\{u$ in $L \mid[u, L] \subset Z(L)\}$ is a characteristic ideal of $L$ containing $V$ and, since $Z(L)=Z(A)$, it is contained in $V+A^{2}$ by hypothesis on $A$. Thus for any $D$ in $\Delta(L), D(V) \subseteq V+A^{2}$. Now let $N=\{D$ in $\Delta(L) \mid D(V) \subseteq A\}$. Then, in fact, the elements of $N$ map $V$ into $A^{2}$ and since $A^{2}=L^{2}$, which is a characteristic ideal of $L, N$ is an ideal of $\Delta(L)$.

If $D$ is in $\Delta(L)$ then $\left(\left.D_{1}\right|_{V}\right)^{*}$ is in $S$ by (2). Thus $D-\left(\left.D_{1}\right|_{V}\right)^{*}$ is a derivation of $L$ and clearly maps $V$ into $A$. Hence $\Delta(L)=S+N$.

To see that $N$ consists of nilpotent derivations, take $D$ in $N$ and see by induction that $D^{k}(A) \subseteq V+A^{2}+\dot{D}_{2}^{k}(A)$, for integral $k \geq 1$. By (1) and the characteristic nilpotence of $A$, there is an $n$ such that $D^{n}(A) \subseteq V+A^{2}$. Then $D^{n+1}(L) \subseteq A^{2}=L^{2}$. Since $L$ is nilpotent, $D$ is nilpotent.

To exhibit a specific $L$ answering 6.1-6.4 take $A$ to be the algebra in [2]. The answer to 6.1 is evidently yes. 6.3 and 6.4 are answered affirmatively since $A$ is directly indecomposable and $\Delta(A)$ annihilates $Z(A)$. $N$ is the maximal nilpotent ideal of $\Delta(L)$ so that to answer 6.2 affirmatively we need only show $[S, N] \neq 0$. for this, let $T_{1}$ and $T_{2}$ in $\Delta(L)$ be defined so that $T_{1} x=0, T_{1} y=z, T_{1}(A)=0$ and $T_{2} x=y, T_{2} y=0, T_{2}(A)=0$. Then $T_{1}$ is in $N, T_{2}$ is in $S$ and $\left[T_{1}, T_{2}\right] \neq 0$.

\section{REFERENCES}

[1] N. Bourbaki, Groupes et Algèbres de Lie, Paris, 1960.

[2] J. Dixmier and W. Lister, Derivations of nilpotent Lie algebras, Proc. A. M. S., vol. 8 (1957), pp. 155-158.

[ 3 ] N. Jacobson, Lie Algebras, New York, 1962.

[ 4 ] G. Leger, Derivations of Lie algebras III, Duke Math. Journ., vol. 30, (1963), pp. 637646.

[ 5 ] E. Schenkman, On the derivation algebra and the holomorph of a nilpotent algebra, Mem. A. M. S., no. 14, (1955), pp. 15-22.

[6] S. Tôgô, On the derivation algebras of Lie algebras, Can. J. Math., vol. 13, (1961), pp. 201-216.

Tufts University

Bucknell University 